\title{
Étude préliminaire sur l'effet dépressif de la molinie (Molinia caerulea) sur la croissance et l'état mycorhizien de semis de chêne rouge (Quercus rubra)
}

\author{
J Timbal 1*, J Gelpe 1, J Garbaye 2 \\ avec la collaboration technique de G Courrier
1 Station INRA de recherches forestières de Bordeaux, laboratoire d'écophysiologie et nutrition, domaine de L'Hermitage, Pierroton, 33610 Cestas;
2 Centre INRA de recherches forestières de Nancy Champenoux, laboratoire de microbiologie forestière, 54280 Seichamps, France

(Reçu le 19 janvier 1990; accepté le 1 octobre 1990)

\begin{abstract}
Résumé - Dans beaucoup de sols acides où il est planté, le chêne rouge d'Amérique est confronté à une graminée sociale très fréquente : la molinie (Molinia caerulea (L) Moench), dont les propriétés allélopathiques ont déjà été mises en évidence (Weise, 1960; Becker et Levy, 1983; Becker, 1984a). On peut émettre l'hypothèse, qu'en plus de la concurrence qu'elle exerce sur les jeunes plants de chêne rouge, la molinie exerce sur eux une action de nature allélopathique, directe ou indirecte, par l'intemédiaire d'une modification défavorable du système mycorhizien (Pélissier et Trosset, 1989). Pour vérifier cette action allélopathique, une première expérimentation en serre a été conduite sur des plants de chêne rouge élevés pendant deux ans dans des conteneurs de grande capacité en présence ou non de molinie. Le substrat de culture est constitué d'un horizon $A_{1}$ sableux, enrichi ou non en engrais, et recevant une alimentation en eau jamais limitante. A la fin de la $2^{\theta}$ saison de végétation, on a comparé la biomasse des tiges et des racines dans les différentes modalités et noté le statut mycorhizien sur un échantillon restreint de celles-ci. On a mis en évidence un effet dépressif très net de la molinie sur la croissance des chênes, accompagné d'une importante modification des mycorhizes : forte régression des mycorhizes à Laccaria (réputées efficaces) et augmentation concomitante des mycorhizes à Cenococcum (réputées peu efficaces), accompagnées d'une nécrose des racines courtes, dans les traitements avec molinie. Ces résultats sont discutés. Ils suggèrent une action de nature allélopathique de la molinie sur les plants de chêne rouge se traduisant en particulier sur le statut mycorhizien. Des expériences complémentaires sont proposées pour confirmer et préciser ces résultats.
\end{abstract}

\section{Quercus rubra / Molinla coerulea / Interférence / allélopathle / mycorhizes / fertilisation}

Summary - Preliminary study of the depressive effect of Mollnia caerulea (L) Moench on early growth and mycorrhizal status of Quercus rubra seedlings. In many acid soils where it is planted, northern red oak (Quercus rubra $L$ ) has to stand the competition of a very common social grass Molinia caerulea (L) Moench. Some allelopathic properties of this species have been shown by different authors (Weise, 1960; Becker et Levy, 1984a). Besides the direct competition of Molinia on young red oak plants, an allelopathic effect can thus be hypothesized, either directly on the plant itself, or indirectly through the symbiotic fungi in the ectomycorrhizas, as shown by Pélissier and

\footnotetext{
* Correspondance et tirés à part.
} 
Trosset (1989) with some fungi associated with Picea abies. In order to verify this hypothesis, a first experiment was performed by growing red oak seedlings for 2 yr in a glasshouse in large containers filled with a sandy soil with the addition or not of mineral fertilizers, alone or with Molinia. Non-limiting water conditions were maintained throughout the experiment. At the end of the second growth season, stem and root biomasses were measured and the mycrorrhizal status of a sub-sample of plant was assessed. The results clearly show a depressive effect of Molinia on the growth of red oak, together with significant modifications in the mycorrhizal status of the roots: regression of ectomycorrhizas formed by Laccaria sp (an efficient fungus) and increasing number of those formed by Cenococcum geophiIum (less efficient) and of non-mycorrhizal and dead short roots. This suggests an indirect allelopathic effect involving the ectomycorrhizal symbiosis. Complementary experiments are proposed in order to confirm and obtain further information regarding these results.

Quercus rubra / Molinia caerulea / interference / allelopathy / fertilization / mycorrhizal status

\section{INTRODUCTION}

En France, dans beaucoup de sols acides où il est planté, le chêne rouge d'Amérique (Quercus rubra $L=Q$ borealis Michx) entre en concurrence durant les premières années avec une végétation adventice où les graminées, du fait de leur comportement héliophile, occupent une place importante, voire exclusive.

Parmi ces graminées adventices, la molinie (Molinia caerulea (L) Moench) est une des plus communes et des plus largement répandues en plaine sur terrain acide et humide.

En plus de la concurrence qu'elle exerce vis-à-vis de l'eau, des éléments nutritifs et parfois de la lumière, elle possède une action allélopathique connue depuis longtemps (Weise, 1960; Becker, 1984a) en particulier vis-à-vis des semis de chêne sessile et de chêne pédonculé (Becker et Levy, 1983).

Aussi, il nous a paru intéressant de vérifier si une action allélopathique pouvait intervenir dans de jeunes plantations de chêne rouge et, si oui, d'essayer d'en mesurer l'impact sur le taux de reprise et la croissance juvénile. On pourrait ainsi expliquer, du moins en partie, les difficultés de reprise rencontrées par les reboiseurs avec cette espèce (Hubert, communication personnelle).

De plus, comme il est possible qu'un effet allélopathique ne s'exerce pas directement sur la plante, mais sur les symbiotes ectomycorhiziens qui constituent l'appareil absorbant (Pélissier et Trosset, 1989; Brown, 1984; Rose et al, 1988), nous avons cherché à mettre en évidence le phénomène.

\section{MATÉRIELS ET MÉTHODES}

Les plants de chêne rouge ont été élevés pendant 2 saisons de végétation en serre à partir de glands semés directement dans des conteneurs de 70 I (5 plants par conteneur), seuls ou en mé. lange avec de la molinie.

Le substrat de culture est constitué d'un horizon $A_{1}$ de podzol sableux landais avec adjonction ou non d'une fertilisation complète apportée en surface, en suspension, dans l'eau sous forme d'ammonitrate, de scories potassiques et de chaux éteinte. Les doses sont les suivantes : $100 \mathrm{~kg} / \mathrm{ha}$ d'azote, sous forme d'ammonitrate (6 g/conteneur, soit $1,2 \mathrm{~g} /$ plant); $150 \mathrm{~kg} / \mathrm{ha}$ de $\mathrm{P}_{2} \mathrm{O}_{5}$, sous forme de scories potassiques $(25 \mathrm{~g} /$ conteneur, soit $5 \mathrm{~g} / \mathrm{plant}$ ); $150 \mathrm{~kg} / \mathrm{ha}$ de $\mathrm{K}_{2} \mathrm{O}$, sous forme de scories potassiques $(25 \mathrm{~g} /$ conteneur, soit $5 \mathrm{~g} /$ plant); $150 \mathrm{~kg} / \mathrm{ha}$ de Ca, sous forme de chaux éteinte (10 $\mathrm{g} /$ conteneur, soit $2 \mathrm{~g}$ /plant). 
Chaque conteneur a reçu 5 glands de chêne rouge disposés régulièrement en surface, avec ou sans 5 éclats (fragment élémentaire de touffe) de molinie repiqués en quinconce entre les glands au moment de la germination.

Les glands de chêne rouge utilisés sont de provenance (artificielle) locale. Ils ont été récoltés sous de vieux chênes rouges du parc du domaine INRA de l'Hermitage, à Cestas (Gironde). Les touffes de molinie, d'où on a extrait les éclats, proviennent également de ce domaine. On a choisi des éclats qui ont des développements aérien et racinaire semblables.

L'expérimentation comporte 4 modalités :

- traitement fertilisation ou non;

- traitement molinie ou non.

Pour chacune de ces modalités on dispose de 6 répétitions : 6 conteneurs de 5 plants, soit 30 plants.

Dans toutes les modalités, pour que l'alimentation en eau ne constitue pas un facteur limitant, les conteneurs baignent par leur base dans de l'eau dont le niveau est régulièrement réajusté. Le substrat de culture étant humidifié au départ (capacité au champ), il n'y a pas ensuite d'apport d'eau en surface.

Bien qu'une influence de l'intensité d'éclairement sur l'action allélopathique de la molinie ait été mise en évidence par Becker (1984b), nous n'avons pas pris en compte ce facteur, voulant nous placer dans les conditions de pleine lumière qui prévalent dans les plantations. Dans notre serre (à couverture plastique assurant une lumière relativement diffuse), les plants cultivés dans des conteneurs reçoivent de la lumière de tous côtés, ce qui leur assure un éclairement non limitant.

A la fin de la $2^{\ominus}$ année de végétation, on a mesuré les biomasses (matières sèches) des plants de chêne rouge après passage à l'étuve à $60^{\circ} \mathrm{C}$ pendant $48 \mathrm{~h}$, ce qui est suffisant pour obtenir un poids constant. II s'agit de la biomasse des tiges (sans les feuilles), de celle des racines et de leur somme. Les biomasses foliaires n'ont pas été mesurées, la chute des feuilles à l'automne étant progressive et la nécessité pour le dispositif d'occuper un espace limité dans la serre empêchant d'identifier avec certitude les plants à l'origine des feuilles tombées. La molinie n'a pas fait l'objet de mesures. Précisons seulement qu'à la fin de l'expérimentation, elle était fructifiée partout et avait atteint une hauteur moyenne semblable à celle des chênes.

La comparaison des résultats entre modalités (moyenne par modalité des moyennes des 5 plants par conteneur) a été faite par analyse de variance (test $F$ au seuil de $5 \%$ ).

En ce qui concerne la mycorhization, les observations ont porté sur un échantillon, à savoir une répétition (5 plants) par modalité. Les modalités avec et sans fertilisation ayant été mélangées, les observations de mycorhizes ont donc porté sur 10 plants de chêne rouge cultivés en présence de molinie et de 10 plants cultivés en l'absence de molinie.

Comme toutes les essences à ectomycorhizes, les chênes ont un système racinaire hiérarchisé : un squelette de racines longues, avec des apex actifs en permanence et pourvus d'une coiffe, qui s'allonge et se ramifie pour explorer le sol, et grossit (développement de structures secondaires). A l'exception des apex en elongation (suçoirs, bouts blancs), ces racines longues se subérisent vite et ont une faible capacité d'absorption. Le système absorbant, caduc, est constitué par les racines courtes portées latéralement par les racines longues. Les racines courtes n'ont pas de coiffe, ont un allongement limité à quelques $\mathrm{mm}$, et sont rapidement transformées en mycorhizes.

Après lavage, 2 racines latérales longues ont été prélevées sur chaque plante : une en haut et une en bas du pivot. Ces 10 racines sont ensuite coupées en tronçons de $5 \mathrm{~cm}$ lesquels sont mélangés, placés dans l'eau et observés avec une loupe binoculaire. Six types de racine courte ont été distingués et notés de 0 à 3 (note d'abondance-répartition) selon l'échelle suivante (Garbaye, 1984) :

0 , pas de mycorhize visible;

1 , rares mycorhizes dispersées;

2, mycorhizes groupées en foyer d'infection;

3, mycorhizes abondantes sur l'ensemble du tronçon observé.

\section{RÉSULTATS}

\section{Biomasses}

Le tableau I donne les valeurs moyennes des biomasses (des tiges sans les feuilles, 
Tableau 1. Biomasse moyenne par modalité (en g de matière sèche).

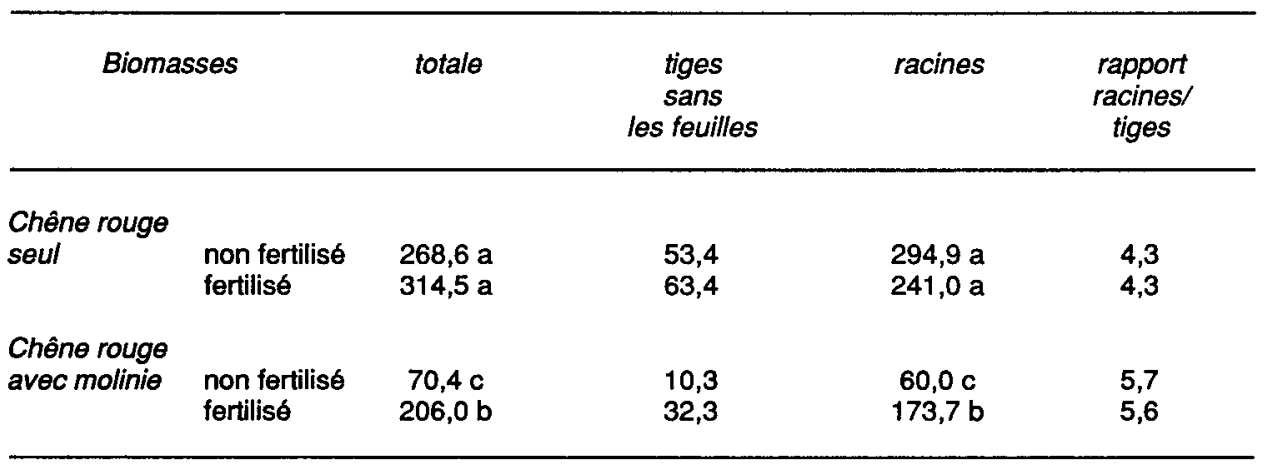

des racines, et de la somme racine + tiges) par modalité ainsi que les rapports biomasse des racines/biomasse des tiges.

Pour les biomasses totales, il apparaît qu'il y a un effet négatif significatif de la molinie, avec ou sans fertilisation, et un effet positif de la fertilisation dans la seule modalité avec molinie.

Pour les biomasses partielles des tiges et des racines, les résultats sont identiques.

Pour le rapport de la biomasse des racines à celle des tiges, la présence de molinie induit une augmentation significative de la biomasse des racines au détriment de celle des tiges, que le substrat soit fertilisé ou non; mais il n'y a pas de différence significative entre les modalités fertilisation ou non, que le chêne rouge pousse seul ou en présence de molinie.

Ces résultats montrent un effet dépressif de la molinie sur la croissance en biomasse des semis de chêne rouge, et un effet fertilisation (positif) sur cette même croissance en biomasse, mais pas sur le rapport de la biomasse des racines à celle des tiges.

\section{Mycorhization}

Le tableau II donne la moyenne des 10 notes affectées, après observation des mycorhizes dans les seuls traitements avec ou sans molinie et donc sans distinction des modalités de fertilisation (à la suite d'une erreur technique).

Le faible échantillon examiné n'a pas permis d'effectuer des tests statistiques sur les notes données.

Qualitativement, les différences de mycorhizes sont importantes : alors que la molinie semble être défavorable au développement des champignons mycorhiziens Scleroderma sp et Laccaria sp, Cenococcum geophilum n'est pas affecté, mais au contraire favorisé.

De plus, on remarque (observations non quantifiées) dans les témoins sans molinie un chevelu racinaire beaucoup plus fourni et des racines courtes plus abondantes par unité de longueur de racine longue, alors que, dans les traitements avec molinie, plus de la moitié des racines courtes sont non mycorhizées et nécrosées. 
Tableau II. Etat mycorhizien des racines courtes (notation de 0 à 3).

$\begin{array}{ccc}\text { Types racines courtes } & \begin{array}{c}\text { Chêne rouge } \\ \text { seul }\end{array} & \begin{array}{c}\text { Chêne rouge } \\ \text { plus molinie }\end{array}\end{array}$

Mycorhizes ramifiées, contournées, à manteau blanc, épais, laineux.

Beaucoup de rhizomorphes blancs poilus (type Scleroderma sp.)

2

Mycorhizes courtes, non ramifiées, à manteau blanc. Beaucoup de mycélium externe, diffus (type A1 de Voiry, dû à Hebeloma sp. Inocybe sp)

Mycorhizes coralloides, à manteau lisse, dense, beige (champignon indéterminé)

Mycorhizes courtes, globuleuses, non ramifiées, à manteau dense, noir, brillant. Hyphes grosses, longues, raides et noires (dues à Cenococcum geophilum)
0 , 9

2

\section{DISCUSSION}

Les paramètres utilisés pour quantifier la croissance des jeunes plants de chêne rouge au bout de leur $2^{\theta}$ saison de végétation (biomasse des tiges (sans les feuilles), des racines, totale, et du rapport racines/ tiges) sont affectés de façon presque semblable par les deux facteurs étudiés : fertilisation et interférence de la molinie.

Sur la croissance en biomasse (totale ou partielle), il y a un effet dépressif de la molinie avec ou sans fertilisation, et un effet positif de la fertilisation seulement en présence de molinie.

Sur le rapport de la biomasse des racines sur celle des tiges, il n'apparaît qu'un effet molinie, se traduisant par une augmentation de la biomasse des racines au détriment de celle des tiges.

L'effet positif de la fertilisation sur la biomasse en présence de molinie montre que la fertilité minérale du substrat ne constitue pas un facteur limitant de la croissance lorsque les semis de chêne rouge poussent seuls (sol nu entre les plants), mais qu'elle le devient en présence de molinie, ce qui suggère un phénomène de concurrence pour les éléments minéraux.

Les différentes valeurs du rapport de la biomasse des racines à celle des tiges est plus difficile à interpréter : l'augmentation significative de ce rapport en présence de molinie, que le sol soit fertilisé ou non, est 
cohérent avec une réduction concomitante de la biomasse, et donc principalement de celle des tiges. Cela suggère une alimentation minérale moins bonne et donc un phénomène de concurrence et/ou une efficacité moindre du système absorbant des chênes : processus de nature plutôt allélopathique.

La simple observation d'un effet dépressif de la molinie n'est donc pas suffisante pour considérer que son action est allélopathique. L'effet dépressif peut traduire une compétition, peut être pour l'eau (mais c'est douteux étant donné que l'alimentation hydrique n'a jamais été limitante) ou, plus vraisemblement, pour l'alimentation minérale.

La présence de molinie réduit aussi l'efficacité absorbante du système mycorhizien des plants de chêne rouge; action négative que l'on peut plus facilement qualifier d'allélopathique. En effet, en présence de molinie, il semble qu'il y ait :

- une réduction du nombre de racines fines et du nombre de racines courtes portées par ces dernières;

- une proportion de racines courtes non mycorhizées qui augmente considérablement;

- des racines courtes beaucoup plus souvent nécrosées, sans que l'on puisse attribuer ces nécroses à une sécheresse édaphique (alimentation en eau non limitante) ou à un phénomène d'hydromorphie (substrat sableux très drainant dans toutes les modalités);

- une modification radicale des types d'ectomycorhize : celles à Scleroderma sp (qui explorent largement le sol grâce à d'abondantes rhizomorphes) et à Laccaria dont l'efficacité est bien reconnue (Le Tacon et al, 1988; Gagon, communication personnelle) faisant place à des mycorhizes à Cenococcum geophilum reconnues peu efficaces en condition hydrique non limi- tante, mais supposées plus résistantes aux stress (Garbaye, non publié).

On peut émettre l'hypothèse que, en présence de molinie, d'une part, ces nécroses de racines courtes et d'autre part, cette modification de l'état mycorhizien sont les symptômes d'une action allélopathique directe de la part de la molinie.

\section{CONCLUSION}

Dans cette première expérience à caractère exploratoir, un effet dépressif très net de la molinie sur la croissance des semis de chêne rouge a pu être mis en évidence.

L'hypothèse retenue est celle d'une réaction à la fois directe (nécrose des racines courtes non mycorhizées et réduction du nombre de racines courtes absorbantes) et indirecte par l'intermédiaire du statut mycorhizien des plants de chêne rouge (réduction de la proportion de racines courtes mycorhizées et remplacement de mycorhizes efficaces à Scleroderma sp et Laccaria sp par des mycorhizes peu efficaces à Cenococcum geophilum), entraînant une alimentation minérale des plants plus difficile, hypothèse en partie de nature allélopathique.

Ces résultats demandent à être confirmés :

- par des observations racinaires permettant la mise en œuvre de test statistiques;

- en distinguant la mycorhization des substrats fertilisés et non fertilisés;

- en rajoutant un témoin molinie seule, avec ou sans fertilisation;

- en étudiant la composition minérale foliaire des plants de chêne rouge dans les différents traitements.

Plusieurs types d'expérimentation pourront aussi apporter des informations supplémentaires : 
- comparaison des effets de la présence de la molinie (plante entière vivante) à ceux de lessivats ou de macérations de certaines de ses parties (feuilles et racines), sur la croissance des semis de chêne rouge et le statut mycorhizien de leurs racines;

- tests in vitro d'extraits (de feuilles ou de racines) de molinie sur des cultures mycéliennes des champignons mycorhiziens concernés.

\section{RÉFÉRENCES}

Becker M (1984a) Propriétés allélopathiques de Molinia caerulea (L) Moench et de Carex brizoides $L$; influence sur la germination et sur la croissance de Lepidium sativum L. Communication au 70 Colloque International sur l'Ecologie, la Biologie et la Systématique des mauvaises herbes, Paris, France

Becker M (1984b) Importance de la lumière dans l'expression des relations allélopathiques en forêt. CR Acad Agric Fr 70, 15451552

Becker M, Levy (1983) Installation et dynamique d'une population de semis de chênes en milieu hydromorphe sous l'influence de divers facteurs (lumière, régime hydrique, compéti- tion herbacée) Acta CEcol CEcol Plant 18, 299-317

Brown RT (1984) Dynamic balance in Pine forest: ground cover-Mycorrhizae-Trees. Proc 6th North Am Conf Mycorrhizal Bend, OR, $277 p$

Garbaye J (1984) Compétitivité des champignons ectomycorhiziens. Rev For Fr (Nancy) 36, 33-43

Le Tacon F, Garbaye M, Bouchard D, Chevalier G, Olivier JM, Guimberteau J, Poitou N, Frochot $H$ (1988) Field results from ectomycorrhizal inoculation in France. In: Canadian Workshop on mycorrhizae in Forestry (Lalonde $\mathrm{M}$ and Piché $\mathrm{Y}$, ed) Université Laval, Québec

Pellissier F, Trosset L (1989) Effect of phytotoxic solutions on the respiration of mycorrhizal and non mycorrhizal spruce roots. (Picea abies L Karst) Ann Sci For 46 (suppl), 731s$733 s$

Rose SL, Perry DA, Pilz D, Schoeneberger MM (1988) Allelopathic effects of litter on the growth and colonization of mycorrhizal fungi. $J$ Chem Ecol 9, 1153-1162

Voiry $H$ (1981) Classifications morphologiques des ectomycorhizes du chêne et du hêtre dans le Nord-Est de la France. Eur J For Pathol 11, 284-289

Weise G (1960) Allelopathic Beeinflüssung und Okotypenbildung bei Molinia caerulea (L) Mench. Ber Schweiz Bot Ges 73, 35 p 